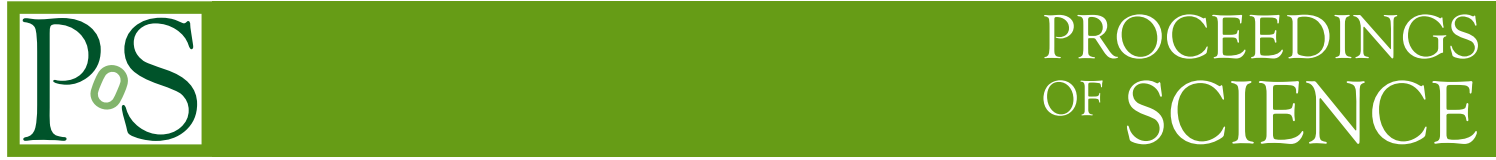

\title{
New bottomonium spectroscopy and transitions
}

\section{Chris West ${ }^{* \dagger}$}

SLAC National Accelerator Laboratory

E-mail: cawesteslac.stanford.edu

Recent results in bottomonium spectroscopy are reviewed. Topics include the observation of $\Upsilon(n S) \rightarrow \eta \Upsilon(1 S)$ transitions, energy scans above the $\Upsilon(4 S)$ resonance by the BABAR and Belle experiments, and the recent observation of the $\eta_{b}$ by the BABAR experiment.

8th Conference Quark Confinement and the Hadron Spectrum September 1-6 2008

Mainz, Germany

${ }^{*}$ Speaker.

${ }^{\dagger}$ A footnote may follow. 


\section{Introduction}

Until recently, the CLEO experiment had the largest data bottomonium data samples, with $22 \times 10^{6} \Upsilon(1 S), 9 \times 10^{6} \Upsilon(2 S)$ and $6 \times 10^{6} \Upsilon(3 S)$ decays. Much larger samples have now been acquired by BABAR with $99 \times 10^{6} \Upsilon(2 S)$ and $122 \times 10^{6} \Upsilon(3 S)$ events and Belle, with $100 \times 10^{6}$ $\Upsilon(1 S)$ and $11 \times 10^{6} \Upsilon(3 S)$ events. In the past year, the BABAR and Belle experiments have also performed scans above the $\Upsilon(4 S)$ resonance. These new datasets have already provided many new results.

\section{Hadronic transitions}

Hadronic transitions between bottomonium states are often described within the QCD multipole expansion (QCDME). If the radius $a$ of a source is smaller than the wavelength of the radiated gluon field, $\lambda \sim 1 / k$, one can expand the gluon field in powers of $a k$. Since the typical radius of a bottomonium state is of order $10^{-1} \mathrm{fm}$ the QCDME is expected to work well for low-lying states. In the charmonium system the ratio $\Gamma(\psi(2 S) \rightarrow \eta J / \psi) / \Gamma\left(\psi(2 S) \rightarrow \pi^{+} \pi^{-} J / \Psi\right)$ as well as the $\pi \pi$ mass spectrum in $\psi(2 S) \rightarrow \pi^{+} \pi^{-} \psi$ transitions are predicted correctly. In the bottomonium system there are many more allowed transitions below open threshold to test the QCDME.

\section{$2.1 \Upsilon(n S) \rightarrow\left(\pi^{0}, \eta\right) \Upsilon(m S)$ transitions}

In the QCDME single $\pi^{0}$ and $\eta$ transitions are suppressed relative to the corresponding dipion transition as the single pseudoscalar transition proceeds via a higher order in the multipole expansion, an $E 1 M 2$ or $M 1 M 1$ transition versus an $E 1 E 1$ transition for the dipion transition.

CLEO has observed for the first time a process involving a b-quark spin flip, supressed due to the large $b$-quark mass, the transition $\Upsilon(2 S) \rightarrow \eta \Upsilon(1 S)$, with a significance of 5.3 standard deviations [1]. The branching fraction for this transition is $\mathscr{B}[\Upsilon(2 S) \rightarrow \eta \Upsilon(1 S)]=\left(2.1_{-0.6}^{+0.7} \pm\right.$ $0.3) \times 10^{-4}$. Related transitions were not observed and upper limits at $90 \%$ confidence level for related processes, in units of $10^{-4}$, are $\mathscr{B}\left[\Upsilon(2 S) \rightarrow \pi^{0} \Upsilon(1 S)\right]<1.8, \mathscr{B}[\Upsilon(3 S) \rightarrow \eta \Upsilon(1 S)]<1.8$, $\mathscr{B}\left[\Upsilon(3 S) \rightarrow \pi^{0} \Upsilon(1 S)\right]<0.7$, and $\mathscr{B}\left[\Upsilon(3 S) \rightarrow \pi^{0} \Upsilon(2 S)\right]<5.1$. The data are presented in fig. 1.

BABAR has searched for $\eta$ transitions between $\Upsilon(m S)(m=4,3,2)$ and $\Upsilon(n S)(n=2,1)$ resonances in an analysis based on $383.2 \times 10^{6} \Upsilon(4 S)$ decays [2]. The $\Upsilon(4 S)$ is produced directly whereas lower $\Upsilon$ initial states are produced via ISR. BABAR has provided the first observation of $\Upsilon(4 S) \rightarrow \eta \Upsilon(1 S)$ decay with a branching fraction $\mathscr{B}[\Upsilon(4 S) \rightarrow \eta \Upsilon(1 S)]=(1.96 \pm 0.06 \pm 0.09) \times$ $10^{-4}$ resulting in the ratio of partial widths $\Gamma(\Upsilon(4 S) \rightarrow \eta \Upsilon(1 S)) / \Gamma\left(\Upsilon(4 S) \rightarrow \pi^{+} \pi^{-} \Upsilon(1 S)\right)=$ $2.41 \pm 0.40 \pm 0.12$. This is larger than the value of $10^{-2}-10^{-3}$ [3] predicted by the QCDME. The data are presented in fig. 1 .

\section{$2.2 \Upsilon(n S) \rightarrow \pi^{+} \pi^{-} \Upsilon(m S)$ transitions}

BABAR also present new measurements of the ratios $\Gamma\left(\Upsilon(4 S) \rightarrow \pi^{+} \pi^{-} \Upsilon(2 S)\right) / \Gamma(\Upsilon(4 S) \rightarrow$ $\left.\pi^{+} \pi^{-} \Upsilon(1 S)\right)=1.16 \pm 0.16 \pm 0.14$ and $\Gamma\left(\Upsilon(3 S) \rightarrow \pi^{+} \pi^{-} \Upsilon(2 S)\right) / \Gamma\left(\Upsilon(3 S) \rightarrow \pi^{+} \pi^{-} \Upsilon(1 S)\right)=0.577 \pm$ $0.026 \pm 0.060$ [2]. While the di-pion mass spectrum in the $4 S \rightarrow 1 S$ transition is in excellent agreement with QCDME predictions, there is a low mass structure in $4 S \rightarrow 2 S$ transitions which is not yet understood. 

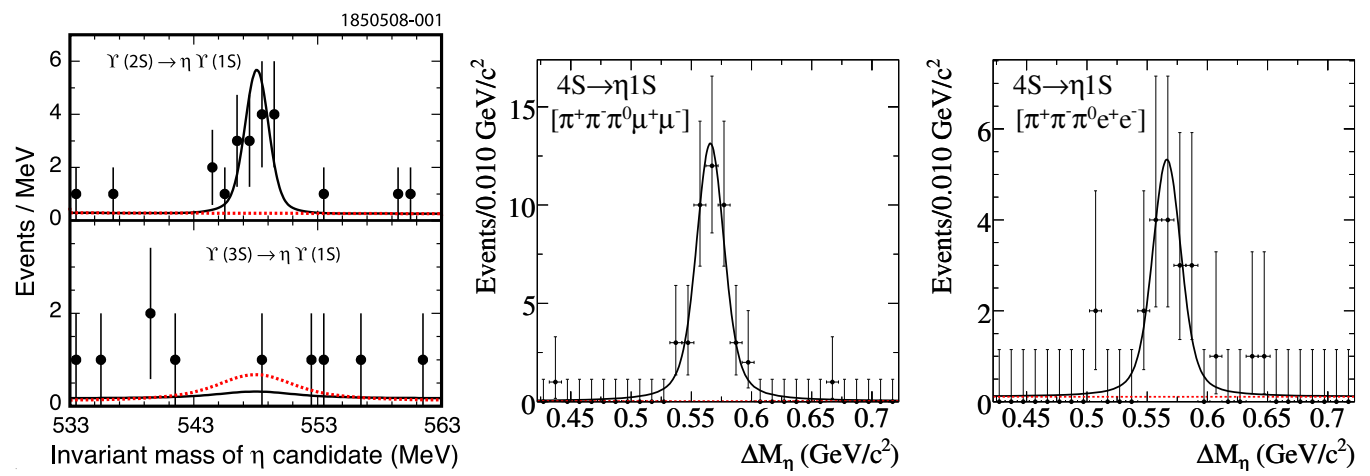

Figure 1: Invariant mass distribution of $\eta$ candidates in kinematically allowed $\Upsilon(3 S) \rightarrow \eta \Upsilon(n S)$ transitions from CLEO (left). Fits to $\Delta M_{\eta}=M_{3 \pi \ell \ell}-M_{\ell \ell}-m_{3 \pi}$ distribution for $\Upsilon(4 S) \rightarrow \eta \Upsilon(1 S)$ candidates with $\Upsilon(1 S) \rightarrow \mu^{+} \mu^{-}$(center) and $\Upsilon(1 S) \rightarrow e^{+} e^{-}$(right) in BABAR $\Upsilon(4 S)$ data.

The Belle experiment has observed unusually large di-pion transition rates of the $\Upsilon(10860)$, with the transition rate $\Upsilon(10860) \rightarrow \pi^{+} \pi^{-} \Upsilon(1 S)$ of $0.59 \mathrm{MeV}$, a factor of $\sim 1000$ larger than the rates of $\Upsilon(n S) \rightarrow \pi^{+} \pi^{-} \Upsilon(1 S)$ of 60,9 , and $19 \mathrm{MeV}$ for $n=2,3$, and 4, respectively [4].

\section{Scans above the $\Upsilon(4 S)$}

Recent observations of exotic charmonium states, as well as the Belle results for anomalous $\Upsilon(10680) \rightarrow \pi^{+} \pi^{-} \Upsilon(n S)$ production, have motivated searches for similar states in the bottomonium system.

Belle has performed a scan of the energy region $\sqrt{s}=10.83 \mathrm{GeV}$ to $\sqrt{s}=11.02 \mathrm{GeV}$ [5]. They observe an enhancement in the $e^{+} e^{-} \rightarrow \pi^{+} \pi^{-} \Upsilon(n S), n=1,2,3$ cross section which is not consistent with the shape of the $\Upsilon(10860)$ and $\Upsilon(11020)$ hadronic cross section. The results are shown in fig. 2.

BABAR has performed a scan of the energy region $10.54 \mathrm{GeV}$ to $11.20 \mathrm{GeV}$ [6], also shown in fig. 2. The interpretation of the results is strongly dependent upon the position of threshold openings $[7,8]$.

\section{Observation of the $\eta_{b}$ at BABAR}

Though the bottomonium system was discovered over thirty years ago, the spin-singlet states have remained elusive. These include the ground state of the bottomonium system, the $\eta_{b}$. Earlier this year the BABAR experiment accumulated $30 \mathrm{fb}^{-1}$ on the $\Upsilon(3 S)$ resonance, where the $\eta_{b}$ may be seen in the hindered $M 1$ radiative decay $\Upsilon(3 S) \rightarrow \gamma \eta_{b}$.

The yield of the signal peak is $19200 \pm 2000 \pm 2100$ events, with a statistical significance, including systematic errors, of $10 \sigma$. The signal peak, after subtracting all backgrounds, is shown in fig. 3. The measured branching fraction of $(4.8 \pm 0.5 \pm 1.2) \times 10^{-4}$ rules out many theoretical predictions of $M 1$ transition rates.

Though neither the spin nor the parity has been measured, the agreement with the theoretical predictions for the $\eta_{b}$ mass, and expected M1 transition rate, leads to an interpretation of the peak 

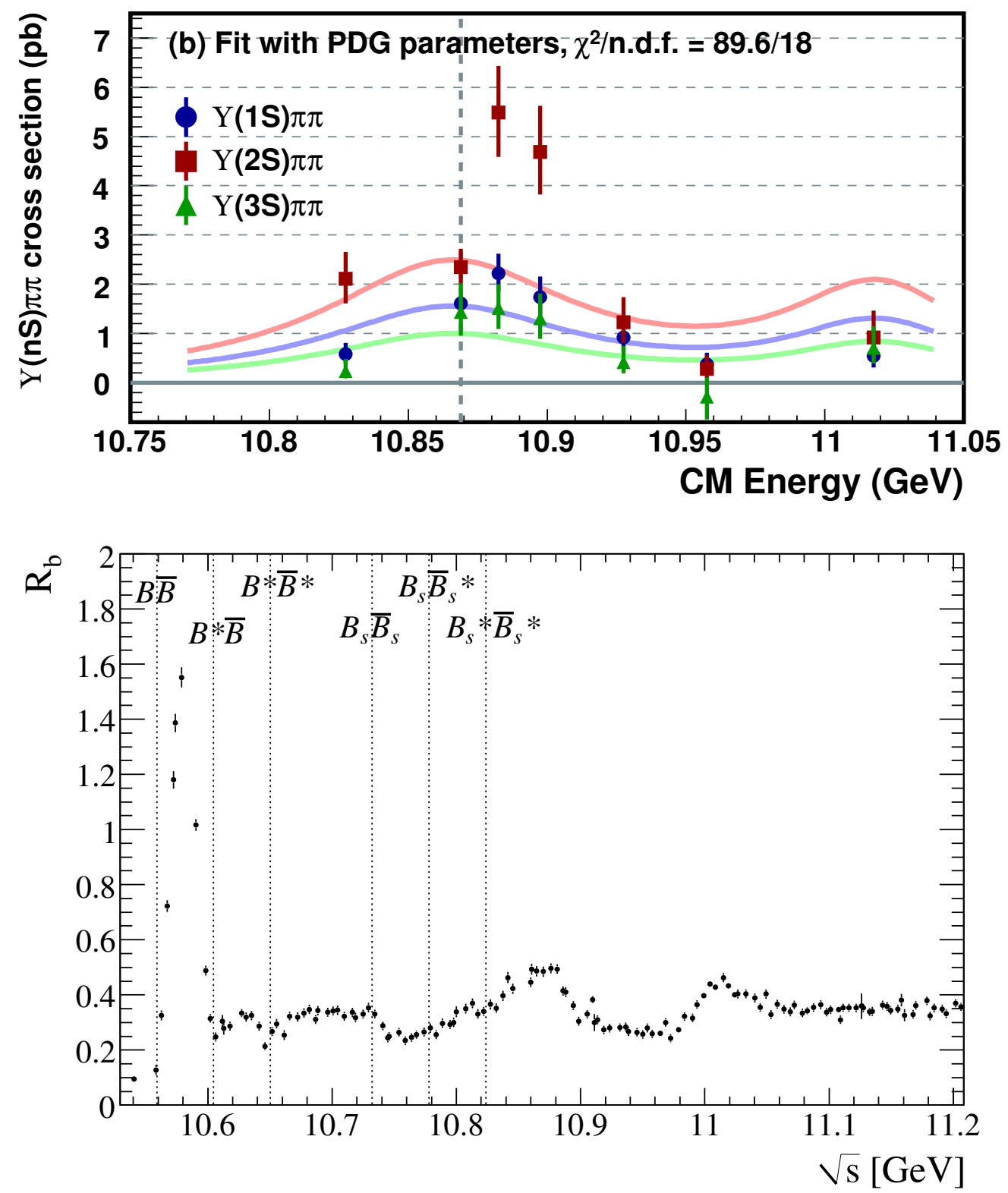

Figure 2: (Top) Production cross section of $e^{+} e^{-} \rightarrow \pi^{+} \pi^{-} \Upsilon(n S), n=1,2,3$ from the Belle experiment. The fit curves describe two non-interfering Breit-Wigner pdfs representing the $\Upsilon(10860)$ and $\Upsilon(11020)$ states on top of a flat background. (Bottom) BABAR scan of the energy region $10.54 \mathrm{GeV}$ to $11.20 \mathrm{GeV}$. The vertical dotted lines mark threshold openings. 


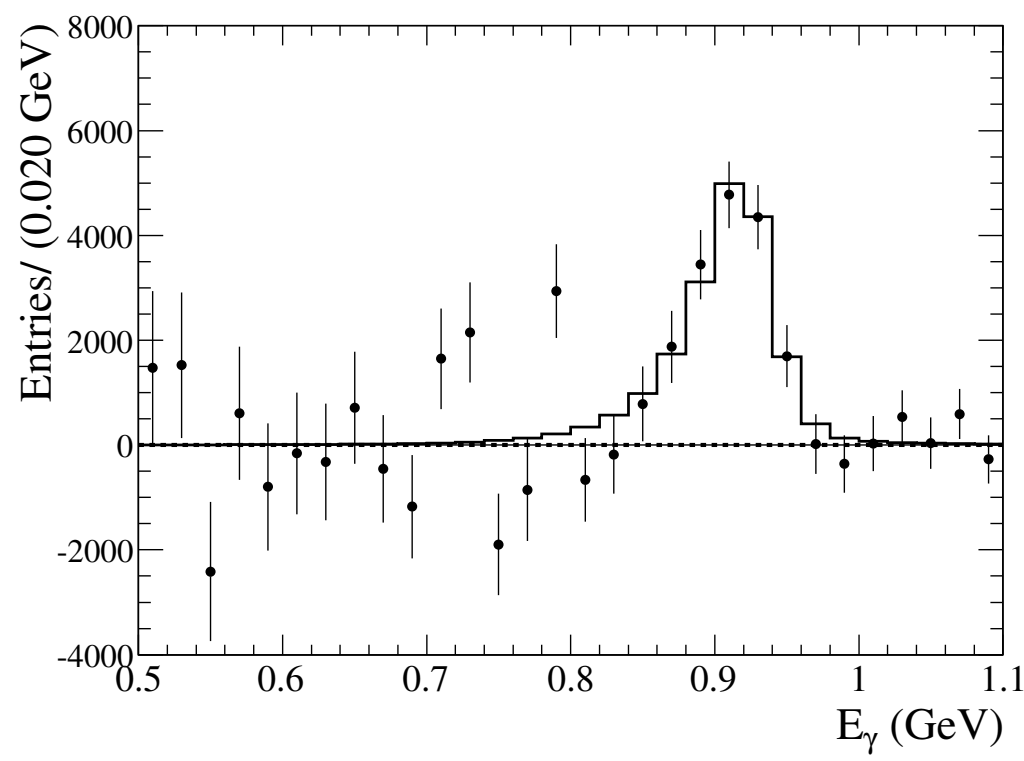

Figure 3: Signal peak for decay $\Upsilon(3 S) \rightarrow \gamma \eta_{b}$ after subtracting all backgrounds, from the BABAR experiment.

as being due to the $\eta_{b}$. Under this interpretation, the mass of the $\eta_{b}$ is $9388.9_{-2.3}^{+3.1} \pm 2.7 \mathrm{MeV} / c^{2}$, corresponding to a hyperfine splitting between the $\eta_{b}$ and $\Upsilon(1 S)$ of $71.4_{-3.1}^{+2.3} \pm 2.7 \mathrm{MeV} / c^{2}$, ruling out many QCD and potential model predictions.

\section{Conclusion}

Recent new data has provided a great deal of new information regarding bottomonium spectroscopy and transitions. The recently acquired data has not yet been fully analyzed. With the much larger datasets currently available, observation of the $\eta_{b}(2 S)$ and the $h_{b}$ should be possible, as well as a confirmation of $\Upsilon(1 D)$ states and precision measurements of electic dipole transition rates.

\section{References}

[1] Q. He et al. (CLEO Collaboration), arXiv:0806.3027v3 [hep-ex]

[2] B. Aubert et al. (BABAR Collaboration), arXiv:0807.2014v1 [hep-ex]

[3] Y. Kuang, Front. Phys. China 119

[4] K.-F. Chen et al. (Belle Collaboration), Phys. Rev. Lett. 100, 112001 (2008)

[5] I. Adachi et al. (Belle Collaboration), arXiv:0808.2445v1 [hep-ex]

[6] B. Aubert et al. (BABAR Collaboration), arXiv:0809.4120v1 [hep-ex]

[7] E. Eichten et al., Phys. Rev. D 17 (1978) 3090; E. Eichten et al., Phys. Rev. D 21 (1980) 203.

[8] N. A. Tornqvist, Phys. Rev. Lett. 53, 878 (1984).

[9] B. Aubert et al. (BABAR Collaboration), Phys. Rev. Lett. 101, 071801 (2008). 